\title{
SOME ASPECTS OF THE LOCAL THEORY OF GENERALIZED DHOMBRES FUNCTIONAL EQUATIONS IN THE COMPLEX DOMAIN
}

\author{
JÖRG TOMASCHEK ${ }^{1}$
}

\begin{abstract}
We study the generalized Dhombres functional equation $f(z f(z))=\varphi(f(z))$ in the complex domain. The function $\varphi$ is given and we are looking for solutions $f$ with $f(0)=w_{0}$ and $w_{0}$ is a primitive root of unity of order $l \geq 2$. All formal solutions for this case are described in this work, for the situation where $\varphi$ can be transformed into a function which is linearizable and local analytic in a neighbourhood of zero we also show that we obtain local analytic solutions. We also discuss an example where it is possible to use other methods than we use in the general case.
\end{abstract}

AMS (2010) subject classification. 39B12, 39B32, 30D05.

Keywords. Generalized Dhombres equation, Iterative functional equation.

Résumé. Nous étudions la fonctionnelle de Dhombres $f(z f(z))=\varphi(f(z))$ dans le plan complexe. La fonction $\varphi$ est donnée et nous cherchons les solutions $f$ avec $f(0)=w_{0}$ où $w_{0}$ est une racine primitive de l'unité d'ordre $l \geq 2$. Nous décrivons dans ce travail toutes les solutions formelles dans ce cas, et lorsque $\varphi$ peut être transformée en une fonction linéarisable et localement analytique au voisinage de zéro nous montrons également comment obtenir des solutions analytiques locales. Nous discutons enfin un exemple où il est possible d'utiliser des méthodes différentes de celles que nous mettons en œuvre dans le cas général.

Mots clefs. Equation de Dhombres généralisée, équation fonctionnelle itérative.

\section{INTRODUCTION}

The generalized Dhombres functional equation in the complex domain is given by

$$
f(z f(z))=\varphi(f(z))
$$

where the function $\varphi$ is a given power series. We are looking for local analytic or formal solutions $f$ of $(1)$ with $f(0)=w_{0}$ where $w_{0}$ is a primitve root of unity of order $l \geq 2$. The generalized Dhombres functional equation was first studied in the complex domain in the year 2005 in [3]. In this paper solutions $f$ with $f(0)=0$ were considered. In 2009 this equation was studied again. This time the interest was focussed on solutions $f$ with $f(0)=w_{0}$ where $w_{0}$ is a complex number which is different from zero and no root of unity.

The original Dhombres equation is given by

$$
f(x f(x))=f(x)^{2}
$$

\footnotetext{
${ }^{1}$ Institute for Mathematics and Scientific Computing, Karl-Franzens-Universität Graz, Heinrichstraße 36, A-8010 Graz, Austria; email: joerg.tomaschek@uni-graz.at.

(C) EDP Sciences, SMAI 2012
} 
where $x$ is a real number. This original equation arised from a population model which was studied by Jean Dhombres (see [1], chapter 6).

The following transformations are part of [4] and these transformations can be done for an arbitrary complex number $w_{0}$ which is different from zero. In a first step (1) becomes by putting $f(z)=w_{0}+g(z), g(0)=0$ equivalent to the transformed generalized Dhombres functional equation

$$
g\left(w_{0} z+z g(z)\right)=\tilde{\varphi}(g(z))
$$

where $g$ is a series given by $g(z)=c_{k} z^{k}+\ldots$ with $c_{k} \neq 0, k \in \mathbb{N}$, and the function $\tilde{\varphi}$ is calculated as $\tilde{\varphi}(\omega)=w_{0}^{k} \omega+d_{2} \omega^{2}+\ldots$. By taking an arbitrary but fixed power series solution $T$ of $T(z)^{k}=g(z)$ we get

$$
T\left(w_{0} T^{-1}(z)+T^{-1}(z) z^{k}\right)=\psi(z)
$$

for the equation above with $\psi(z)^{k}=\tilde{\varphi}\left(z^{k}\right)$. Finally defining $U=T^{-1}$ leads to the linear functional equation

$$
\left(w_{0}+z^{k}\right) U(z)=U(\psi(z))
$$

The function $\varphi$ in equation (1) is known and so it is possible to compute $\psi$, we get $\psi(z)=w_{0} z\left(1+\frac{d_{2}}{w_{0}^{k} k} z^{k}+\ldots\right)$. From now on we suppose that $w_{0}$ is a primitive root of unity of order $l \geq 2$.

\section{Generalized Dhombres functional EQuation with a Root of unity $w_{0}$}

In this section we want to give a full description of all formal solutions of equation (1) with $f(0)=w_{0}$ and $w_{0}$ is a primitive root of unity of order $l \geq 2$. The case where $w_{0}=1$ has to be treated seperately. We start with a first Lemma but before we note that equation (3) is called solvable, if it has a solution $U$ which is different from zero.

Lemma 1.1. If (3) is solvable for given $\psi$ and $k$ then there exists a solution $U_{0}$ with $U_{0}(z) \in z \mathbb{C} \llbracket z^{k} \rrbracket \cap \Gamma_{1}$.

Proof. We write $U(z)=u_{1} z U^{\star}(z)$ with $U^{\star}(z)=1+\ldots$, then (3) is equivalent to

$$
\frac{w_{0} z\left(1+w_{0}^{-1} z^{k}\right)}{\psi(z)} U^{\star}(z)=U^{\star}(\psi(z)) .
$$

The series above on the left hand side starts with 1 and hence one can introduce the formal logarithm. Then using the functional equation for the formal logarithm we get

$$
\operatorname{Ln} \frac{w_{0} z}{\psi(z)}+\operatorname{Ln}\left(1+w_{0}^{-1} z^{k}\right)=X^{\star}(\psi(z))-X^{\star}(z)
$$

with $X^{\star}=\operatorname{Ln} U^{\star}$ and ord $X^{\star}>0$. For the function $\psi$ it follows from the assumption $\psi(z)^{k}=\tilde{\varphi}\left(z^{k}\right)$ that $\psi(z) \in z \mathbb{C} \llbracket z^{k} \rrbracket \cap \Gamma$ and hence the left side $\operatorname{Ln} \frac{w_{0} z}{\psi(z)}+\operatorname{Ln}\left(1+w_{0}^{-1} z^{k}\right)$ of $(4)$ belongs to $\mathbb{C} \llbracket z^{k} \rrbracket$. It is possible to write

for $X^{\star}$. Then we get

$$
X^{\star}(y)=\sum_{\substack{\nu \geq 1 \\ \nu \neq \equiv(\bmod k)}} \xi_{\nu}^{\star} y^{\nu}+\sum_{\substack{\nu \geq 1 \\ \nu \equiv 0(\bmod k)}} \xi_{\nu}^{\star} y^{\nu}
$$

$$
X^{\star}(\psi(z))-X^{\star}(z)=\sum_{\substack{\nu \geq 1 \\ \nu \neq \equiv(\bmod k)}} \xi_{\nu}^{\star}\left(\psi(z)^{\nu}-z^{\nu}\right)+\sum_{\substack{\nu \geq 1 \\ \nu \equiv 0(\bmod k)}} \xi_{\nu}^{\star}\left(\psi(z)^{\nu}-z^{\nu}\right)
$$


where the first part $\sum_{\substack{\nu \geq 1 \\ \nu \neq 0(\bmod k)}} \xi_{\nu}^{\star}\left(\psi(z)^{\nu}-z^{\nu}\right)$ does not contain any term $z^{\nu}$ with $\nu \equiv 0(\bmod k)$ while the

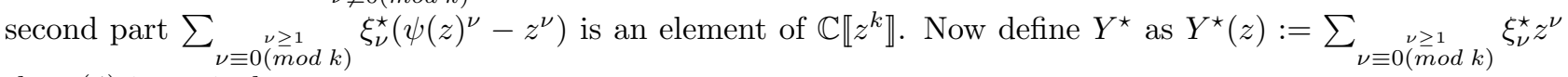
then (4) is equivalent to

$$
\operatorname{Ln} \frac{w_{0} z}{\psi(z)}+\operatorname{Ln}\left(1+w_{0}^{-1} z^{k}\right)=Y^{\star}(\psi(z))-Y^{\star}(z)
$$

and hence (4) has a solution $Y^{\star} \in \mathbb{C} \llbracket z^{k} \rrbracket$ and after reversing our calculations we obtain a function $U_{0}$ with the desired property.

So we know the structure of a solution $U$ with $U(z)=u_{1} U_{0}(z)$ and $U_{0} \in z \mathbb{C} \llbracket z^{k} \rrbracket \cap \Gamma_{1}$ of (3). Next we have to show that there exists a solution. Therefore we have to do some transformations. Like in the proof above we have the equation

$$
w_{0} z \frac{\left(1+w_{0}^{-1} z^{k}\right)}{\psi(z)} U^{\star}(z)=U^{\star}(\psi(z)) .
$$

Define $\hat{U}$ by $U^{\star}(z)^{k}=\hat{U}(z)$, then $\hat{U}$ is given by $\hat{U}(z)=1+\ldots$ and conversely $U^{\star}$ is uniquely determined by $\hat{U}$. Taking the $k-t h$ power and using $U^{\star}(z)^{k}=\hat{U}(z)$ in the equation above leads to

$$
\frac{w_{0}^{k} z^{k}\left(1+w_{0}^{-1} z^{k}\right)^{k}}{\psi(z)^{k}} \hat{U}(z)=\hat{U}(\psi(z))
$$

and again with the formal logarithm this becomes equivalent to

$$
\operatorname{Ln} \frac{w_{0}^{k} z^{k}\left(1+w_{0}^{-1} z^{k}\right)^{k}}{\psi(z)^{k}}=\hat{X}(\psi(z))-\hat{X}(z)
$$

with $\hat{X}:=\operatorname{Ln} \hat{U}$. Now as a consequence of $\psi(z) \in z \mathbb{C} \llbracket z^{k} \rrbracket$ and some other computations it is possible to write $\hat{X}(z)=\tilde{X}\left(z^{k}\right)$ and therefore with $y=z^{k}$ and $\omega_{0}:=w_{0}^{k}$ we get

$$
\operatorname{Ln} \frac{\omega_{0} y\left(1+w_{0}^{-1} y\right)^{k}}{\tilde{\varphi}(y)}=\tilde{X}(\tilde{\varphi}(y))-\tilde{X}(y)
$$

and this leads to

$$
\operatorname{Ln} \frac{\omega_{0} y}{\tilde{\varphi}(y)}+k \operatorname{Ln}\left(1+w_{0}^{-1} y\right)=\tilde{X}(\tilde{\varphi}(y))-\tilde{X}(y)
$$

where $\omega_{0}$ is again a root of unity of order $l_{1}$ and $l_{1}=\frac{l}{\operatorname{gcd}(k, l)}$. Now it is necessary to distinguish two cases. The first case deals with a function $\tilde{\varphi}$ which is not linearizable and hence the function is conjugated to a non linear semicanonical form. So $\tilde{\varphi}$ can be written as

$$
\tilde{\varphi}(y)=\left(R^{-1} \circ N \circ R\right)(y)
$$

with $N(y)=\omega_{0} y+\delta_{n l_{1}+1} y^{n l_{1}+1}+\ldots$ and $\delta_{n l_{1}+1} \neq 0$. The function $R^{-1}$ and hence also the function $R$ is uniquely determined by $R^{-1}(y)=y+\rho_{2} y^{2}+\ldots$ and the condition that the coefficients $\rho_{\mu l_{1}+1}=0$ for $\mu \geq 1$. The number $l_{1}$ denotes the order of $\omega_{0}$. Then (5) is equivalent to

$$
\operatorname{Ln} \frac{\omega_{0} y}{\tilde{\varphi}(y)}+k \operatorname{Ln}\left(1+w_{0}^{-1} y\right)=\tilde{X}\left(R^{-1}(N(R(y)))\right)-\tilde{X}(y)
$$

and substituting $R^{-1}(y)$ for $y$ and defining $X$ as $X:=\tilde{X} \circ R^{-1}$ leads to

$$
\operatorname{Ln} \frac{\omega_{0} R^{-1}(y)}{R^{-1}(N(y))}+k \operatorname{Ln}\left(1+w_{0}^{-1} R^{-1}(y)\right)=X(N(y))-X(y) .
$$


Discussing the right hand side $X(N(y))-X(y)$ of equation (6) in detail leads to the fact that the first coefficient of $X(N(y))-X(y)$ which is different from zero is the coefficient which belongs to the term $y^{(n+1) l_{1}}$. So we have

Lemma 1.2. Equation (6) has a solution if and only if the coefficients of $y^{l_{1}}, \ldots, y^{n l_{1}}$ of the left hand side of equation (6) are zero.

Proof. We write

$$
\frac{\omega_{0} R^{-1}(y)}{R^{-1}(N(y))}=\frac{\frac{\omega_{0} R^{-1}(y)}{\omega_{0} y}}{\frac{R^{-1}(N(y))}{N(y)}} \frac{\omega_{0} y}{N(y)}
$$

and therefore

$$
\begin{aligned}
\operatorname{Ln} \frac{\omega_{0} R^{-1}(y)}{R^{-1}(N(y))} & =\operatorname{Ln} \frac{R^{-1}(y)}{y}-\operatorname{Ln} \frac{R^{-1}(N(y))}{N(y)}-\operatorname{Ln} \frac{N(y)}{\omega_{0} y} \\
& =\operatorname{Ln} \frac{R^{-1}(y)}{y}-\left.\operatorname{Ln} \frac{R^{-1}(u)}{u}\right|_{u=N(y)}-\operatorname{Ln} \frac{N(y)}{\omega_{0} y} .
\end{aligned}
$$

Then we obtain

$$
\frac{N(y)}{\omega_{0} y}=\frac{1}{\omega_{0} y}\left(\omega_{0} y+\delta_{n l_{1}+1} y^{n l_{1}+1}+\ldots\right)=1+\omega_{0}^{-1} \delta_{n l_{1}+1} y^{n l_{1}}+\ldots \in \mathbb{C} \llbracket y^{l_{1}} \rrbracket
$$

where $\omega_{0}^{-1} \delta_{n l_{1}+1} \neq 0$ and hence

$$
\operatorname{Ln} \frac{N(y)}{\omega_{0} y}=\omega_{0}^{-1} \delta_{n l_{1}+1} y^{n l_{1}}+\ldots
$$

Therefore it is only necessary to investigate the coefficients of $y^{l_{1}}, \ldots, y^{(n-1) l_{1}}$ of $\operatorname{Ln} \frac{R^{-1}(y)}{y}-\left.\operatorname{Ln} \frac{R^{-1}(u)}{u}\right|_{u=N(y)}$. Hence we get

$$
\operatorname{Ln} \frac{R^{-1}(y)}{y}=\tilde{\rho}_{2} y+\ldots+\tilde{\rho}_{l_{1}} y^{l_{1}-1}+\tilde{\rho}_{l_{1}+1} y^{l_{1}}+\ldots
$$

with polynomials $\tilde{\rho}_{\mu}$ which depend on $\left(\rho_{2}, \ldots, \rho_{\mu}\right)$ for $\mu \not \equiv 1\left(\bmod l_{1}\right)$ and on $\left(\rho_{2}, \ldots, \rho_{\mu-1}\right)$ for $\mu \equiv 1\left(\bmod l_{1}\right)$. We write

and so we achieve

$$
\operatorname{Ln} \frac{R^{-1}(y)}{y}=: \Theta(y)=\theta_{1} y+\theta_{2} y^{2}+\ldots
$$

$$
\begin{aligned}
\left.\operatorname{Ln} \frac{R^{-1}(u)}{u}\right|_{u=N(y)} & =\Theta(N(y))=\theta_{1}\left(\omega_{0} y+\delta_{n l_{1}+1} y^{n l_{1}+1}+\ldots\right)+\theta_{2}\left(\omega_{0} y+\delta_{n l_{1}+1} y^{n l_{1}+1}+\ldots\right)^{2}+\ldots \\
& =\theta_{1} \omega_{0} y+\theta_{2} \omega_{0}^{2} y^{2}+\ldots+\theta_{l_{1}-1} \omega_{0}^{l_{1}-1} y^{l_{1}-1}+\theta_{l_{1}} y^{l_{1}}+\ldots+\theta_{2 l_{1}} y^{2 l_{1}}+\ldots+\theta_{n l_{1}} y^{n l_{1}}+\ldots \\
& \equiv \theta_{1} \omega_{0} y+\theta_{2} \omega_{0}^{2} y^{2}+\ldots \quad\left(\bmod \text { ord } n l_{1}+1\right) \\
& \equiv \Theta\left(\omega_{0} y\right) \quad\left(\bmod \text { ord } n l_{1}+1\right)
\end{aligned}
$$

and hence $\Theta(y)-\Theta(N(y)) \equiv \Theta(y)-\Theta\left(\omega_{0} y\right)\left(\bmod\right.$ ord $\left.n l_{1}+1\right)$ and therefore there are no coefficients different from zero for $y^{l_{1}}, \ldots, y^{n l_{1}}$ in $\operatorname{Ln} \frac{R^{-1}(y)}{y}-\left.\operatorname{Ln} \frac{R^{-1}(u)}{u}\right|_{u=N(y)}$. It remains to determine the coefficients of $k \operatorname{Ln}(1+$ $\left.w_{0}^{-1} R^{-1}(y)\right)$. Computing $k \operatorname{Ln}\left(1+w_{0}^{-1} R^{-1}(y)\right)$ it is clear that comparing the coefficients of $y^{l_{1}}$ leads to

$$
\rho_{l_{1}}=P_{l_{1}}\left(\rho_{\alpha} \mid \alpha<l_{1}\right) \text {. }
$$

with a polynomial $P_{l_{1}}$. Proceeding in comparing the coefficients of $y^{\mu l_{1}}$ with $\mu \geq 2$ leads to

$$
\rho_{\mu l_{1}}=P_{\mu l_{1}}\left(\rho_{\alpha} \mid \alpha<\mu l_{1}\right)
$$


with polynomials $P_{\mu l_{1}}$ for $2 \leq \mu \leq(n-1) l_{1}$. For $y^{n l_{1}}$ we obtain

$$
-\omega_{0}^{-1} \delta_{n l_{1}+1}+k w_{0}^{-1} \rho_{n l_{1}}+P_{n l_{1}}\left(\rho_{\alpha} \mid \alpha<n l_{1}\right)=0
$$

with a polynomial $P_{n l_{1}}$ and hence

$$
\delta_{n l_{1}+1}=k \omega_{0} w_{0}^{-1} \rho_{n l_{1}}+\omega_{0} P_{n l_{1}}\left(\rho_{\alpha} \mid \alpha<n l_{1}\right)
$$

Now one obtains $\delta_{n l_{1}+1} \neq 0$ if $\rho_{n l_{1}}$ is suitably choosen.

It is also possible to determine the number $k$ if $N$ and $R$ and hence $\tilde{\varphi}$ are given. We get

$$
k=\frac{\delta_{n l_{1}+1}-\omega_{0} P_{n l_{1}}\left(\rho_{\alpha} \mid \alpha<n l_{1}\right)}{\omega_{0} w_{0}^{-1} \rho_{n l_{1}}} .
$$

In summary we achieve $(n-1) l_{1}$ algebraic equations

$$
\rho_{\mu l_{1}}=P_{\mu l_{1}}\left(\rho_{\alpha} \mid \alpha<\mu l_{1}\right)
$$

for $1 \leq \mu \leq(n-1) l_{1}$ and one additional equation

$$
-\omega_{0}^{-1} \delta_{n l_{1}+1}+k w_{0}^{-1} \rho_{n l_{1}}+P_{n l_{1}}\left(\rho_{\alpha} \mid \alpha<n l_{1}\right)=0 .
$$

Theorem 1.3. Let $k \in \mathbb{N}$ be given by (7) and let $\tilde{\varphi}$ be not linearizable and be given by $\tilde{\varphi}(y)=R^{-1}(N(R(y)))$ where $N$ denotes the semicanonical form of $\tilde{\varphi}$. Then the equation

$$
\operatorname{Ln} \frac{\omega_{0} R^{-1}(y)}{R^{-1}(N(y))}+k \operatorname{Ln}\left(1+w_{0}^{-1} R^{-1}(y)\right)=X(N(y))-X(y)
$$

has a solution $X$ if and only if the polynomial relations (8) and (9) are fulfilled. This solution $X$ is unique.

Proof. We obtain the uniquely determined solution if we compare the coefficients of $(6)$ namley $\operatorname{Ln} \frac{\omega_{0} R^{-1}(y)}{R^{-1}(N(y))}+$ $k \operatorname{Ln}\left(1+w_{0}^{-1} R^{-1}(y)\right)=X(N(y))-X(y)$. It is sufficient to look at $\operatorname{Ln} \frac{\omega_{0} R^{-1}(y)}{R^{-1}(N(y))}+k \operatorname{Ln}\left(1+w_{0}^{-1} R^{-1}(y)\right)=$ $\hat{\theta}_{1} y+\hat{\theta}_{2} y^{2}+\ldots$ Let the series $X$ be given by $X(y)=\tilde{\beta}_{1} y+\tilde{\beta}_{2} y^{2}+\ldots$, then we obtain $\tilde{\beta}_{\mu}=\left(\omega_{0}^{\mu}-1\right)^{-1} \hat{\theta}_{\mu}$ for $1 \leq \mu \leq l_{1}-1$. Comparing the coefficients of $y^{l_{1}}$ leads to the first polynomial relation of (8) but it is not possible to determine $\tilde{\beta}_{l_{1}}$ from this equation. Proceeding we can compute the coefficients $\tilde{\beta}_{l_{1}+1}, \ldots, \tilde{\beta}_{2 l_{1}-1}$ and for $y^{2 l_{1}}$ we obtain the second polynomial relation of (8). These steps can be done till we compute the coefficient of $y^{(n+1) l_{1}}$ because there we get $\tilde{\beta}_{l_{1}}=\left(l_{1} \omega_{0}^{l_{1}-1} \delta_{n l_{1}+1}\right)^{-1} \hat{\theta}_{(n+1) l_{1}+1}$. Inductively we obtain the uniquely determined solution $X$ of $(6)$.

Remark 1.4. It is possible to go back the way from $X$ to the function $U$ and hence we get also the solution $U$ of (3). In more detail we have $\tilde{X}(y)=X(R(y))$ and therefore $\hat{X}(y)=\tilde{X}\left(y^{k}\right)=X\left(R\left(y^{k}\right)\right.$. This leads to $\hat{U}(y)=\exp \left(X\left(R\left(y^{k}\right)\right)\right)$. Next we obtain $U(y)=u_{1} z\left[\exp \left(X\left(R\left(y^{k}\right)\right)\right)\right]^{\frac{1}{k}}$. Then we get $T(y)=$ $\left(u_{1} z\left[\exp \left(X\left(R\left(y^{k}\right)\right)\right)\right]^{\frac{1}{k}}\right)^{[-1]}$ and finally $g$ is given by

$$
g(y)=\left\{\left(u_{1} z\left[\exp \left(X\left(R\left(z^{k^{2}}\right)\right)\right)\right]^{\frac{1}{k}}\right)^{[-1]}\right\}^{\frac{1}{k}}
$$


The solutions $f$ of the generalized Dhombres functional equation are given by

$$
f(z)=w_{0}+\left\{\left(u_{1} z\left[\exp \left(X\left(R\left(z^{k^{2}}\right)\right)\right)\right]^{\frac{1}{k}}\right)^{[-1]}\right\}^{\frac{1}{k}} .
$$

This remark also holds for the following theorem, namely Theorem 1.6. That means also in 1.6 we can reverse the calculations like we do in 1.4 and finally we get solutions of the form (10). The difference is that in the case of Theorem 1.3 the solution $X$ is uniquely determined whereas in the situation of Theorem 1.6 the solution $X$ is depending on some arbitrary coefficients.

But before we prove the next theorem we have to consider the following remark.

Remark 1.5. Let $w_{0}$ be a primitive root of unity of order $l \geq 1, k \in \mathbb{N}$ and $\omega_{0}$ be defined by $\omega_{0}=w_{0}^{k}$ where the order of $\omega_{0}$ equals $l_{1}$. Also the series $N$ is given by $N(y)=\omega_{0} y+\delta_{n l_{1}+1} y^{n l_{1}+1}+\ldots \in y \mathbb{C} \llbracket y^{l_{1}} \rrbracket$ with $n \geq 1$ and the coefficient $\delta_{n l_{1}+1}$ is different from zero. Then let the series $H$ be given by $H(y)=h_{1} y+h_{2} y^{2}+\ldots \in \mathbb{C} \llbracket y \rrbracket$ such that the coefficients $h_{\mu l_{1}}$ of $H$ with $1 \leq \mu \leq(n-1) l_{1}$ are given by the polynomial relations

$$
h_{\mu l_{1}}=P_{\mu l_{1}}\left(h_{\alpha} \mid \alpha<\mu l_{1}\right)
$$

where the polynomials $P_{\mu l_{1}}$ are the same as in (8) and such that the coefficients $h_{\mu l_{1}+1}$ are zero for $\mu \geq 1$ and

$$
\left(H^{-1} \circ N \circ H\right)(y)=\omega_{0} y+\ldots \in \mathbb{C} \llbracket y \rrbracket .
$$

The coeffiecient $h_{n l_{1}}$ has to be choosen such that

$$
k=\frac{\delta_{n l_{1}+1}-\omega_{0} P_{n l_{1}}\left(h_{\alpha} \mid \alpha<n l_{1}\right)}{\omega_{0} w_{0}^{-1} h_{n l_{1}}}
$$

holds. Then for every choice of the coefficients of the series $N$ except the coefficient $\delta_{n l_{1}+1}$ and for every choice of the coefficients of the series $H$ except these coefficients we get from the polynomial relations and also except the coefficient $h_{n l_{1}}$ there exists a unique solution $f$ of the generalized Dhombres functional equation

$$
f(z f(z))=w_{0}+H^{-1}(N(H(f(z)))) .
$$

Now we have to consider the second situation, this case deals with a linearizable $\tilde{\varphi}$. Then there exists a function $\tilde{R}$ such that $\tilde{\varphi}(y)=\tilde{R}^{-1}\left(\omega_{0} \tilde{R}(y)\right)$. But it is also possible to use the notations from the case where a non linear semicanonical form $N$ exists. We may consider the present situation as a limiting case of the previous situation for $n \rightarrow \infty$.

In formula (8) $\rho_{\mu l_{1}}=P_{\mu l_{1}}\left(\rho_{\alpha} \mid \alpha<\mu l_{1}\right)$ exactly $(n-1) l_{1}$ algebraic equations as a consequence of the non linear semicanonical form are given. If now the semicanonical form $N(y)=\omega_{0} y+\delta_{n l_{1}+1} y^{n l_{1}+1}+\ldots$ with $\delta_{n l_{1}+1} \neq 0$ becomes linear that means $n \rightarrow \infty$ then we obtain infinitely many algebraic equations instead of finitely many ones. Therefore we get the algebraic relations

for $\mu \geq 1$.

$$
\rho_{\mu l_{1}}=P_{\mu l_{1}}\left(\rho_{\alpha} \mid \alpha<\mu l_{1}\right)
$$

Theorem 1.6. Let $k \in \mathbb{N}$ and $\tilde{\varphi}$ be linearizable and given by $\tilde{\varphi}(y)=R^{-1}\left(\omega_{0} R(y)\right)$. Then the equation

$$
\operatorname{Ln} \frac{\omega_{0} R^{-1}(y)}{R^{-1}\left(\omega_{0} y\right)}+k \operatorname{Ln}\left(1+w_{0}^{-1} R^{-1}(y)\right)=X\left(\omega_{0} y\right)-X(y)
$$

has infinitely many solutions $X$ if the polynomial relations (11) are fulfilled. 
Proof. We consider equation (6) namely

$$
\operatorname{Ln} \frac{\omega_{0} R^{-1}(y)}{R^{-1}\left(\omega_{0}\right)}+k \operatorname{Ln}\left(1+w_{0}^{-1} R^{-1}(y)\right)=X\left(\omega_{0} y\right)-X(y)
$$

and again it is possible to write $\operatorname{Ln} \frac{\omega_{0} R^{-1}(y)}{R^{-1}\left(\omega_{0}\right)}+k \operatorname{Ln}\left(1+w_{0}^{-1} R^{-1}(y)\right)=\tilde{\rho}_{1} y+\tilde{\rho}_{2} y^{2}+\ldots$ Let $X(y)=\beta_{1} y+\beta_{2} y^{2}+\ldots$, then

$$
X\left(\omega_{0} y\right)-X(y)=\sum_{\substack{\nu \geq 1 \\ \nu \neq 0\left(\bmod l_{1}\right)}} \beta_{\nu}\left(\omega_{0}^{\nu}-1\right) y^{\nu}
$$

and therefore we obtain a special solution $X_{0}$ which is given by

$$
X_{0}(y)=\sum_{\substack{\nu \geq 1 \\ \nu \neq 0\left(\bmod l_{1}\right)}} \tilde{\rho}_{\nu}\left(\omega_{0}^{\nu}-1\right)^{-1} y^{\nu}
$$

Hence the general solution is given by

$$
X(y)=\sum_{\substack{\nu \geq 1 \\ \nu \neq 0\left(\bmod l_{1}\right)}} \tilde{\rho}_{\nu}\left(\omega_{0}^{\nu}-1\right)^{-1} y^{\nu}+\sum_{\nu \geq 1} \beta_{\nu l_{1}} y^{\nu l_{1}}
$$

with arbitrary $\beta_{\nu l_{1}}$ for $\nu \geq 1$.

Remark 1.7. Another representation of the solution $U$ of $\left(w_{0}+z^{k}\right) U(z)=U(\psi(z))$ where $\psi$ is linearizable and $w_{0}$ is a root of unity of order $l \geq 2$ can be found in [2] Theorem 5 p. 90.

\section{A Class of EXAMPles}

In this section we want to consider an example of a solution of the generalized Dhombres functional equation where the function $\tilde{\varphi}$ is linearizable. This example is very interesting because we compute it in contrast to the previous section without the use of the formal logarithm. Therefore again one considers $(3)\left(w_{0}+z^{k}\right) U(z)=$ $U(\psi(z))$ where the function $\psi$ is linearizable. Notice that the function $\tilde{\varphi}$ is linearizable if and only if the function $\psi$ is. Let $w_{0}$ be a root of unity with order $l \geq 2$. Then we know that $\omega_{0}:=w_{0}^{k}$ is a root of unity with order $l_{1}=\frac{l}{\operatorname{gcd}(k, l)}$.

We iterate equation (3) and so we obtain

$$
\left(w_{0}+\psi(z)^{k}\right) U(\psi(z))=U\left(\psi^{2}(z)\right)
$$

and hence

$$
\left(w_{0}+\tilde{\varphi}\left(z^{k}\right)\right)\left(w_{0}+z^{k}\right) U(z)=U\left(\psi^{2}(z)\right) .
$$

Iterating the last equation $l_{1}-2$ times and using $\psi(z)^{k}=\tilde{\varphi}\left(z^{k}\right)$ as well as $y=z^{k}$ and $w_{0}^{l}=1$ leads to the equation

$$
\left(1+w_{0}^{-1} \tilde{\varphi}^{l-1}(y)\right)\left(1+w_{0}^{-1} \tilde{\varphi}^{l-2}(y)\right) \ldots\left(1+w_{0}^{-1} \tilde{\varphi}(y)\right)\left(1+w_{0}^{-1} y\right)=1 .
$$

Then there exists a $p \in \mathbb{N}$ such that $l=l_{1} p$ and so as a consequence of $\tilde{\varphi}^{l_{1}}(y)=y$ we obtain

$$
\left(1+w_{0}^{-1} \tilde{\varphi}^{p l_{1}-1}(y)\right)\left(1+w_{0}^{-1} \tilde{\varphi}^{p l_{1}-2}(y)\right) \ldots\left(1+w_{0}^{-1} \tilde{\varphi}(y)\right)\left(1+w_{0}^{-1} y\right)=1
$$

which becomes

$$
\left(1+w_{0}^{-1} \tilde{\varphi}^{l_{1}-1}(y)\right)\left(1+w_{0}^{-1} \tilde{\varphi}^{l_{1}-2}(y)\right) \ldots\left(1+w_{0}^{-1} \tilde{\varphi}(y)\right)\left(1+w_{0}^{-1} y\right)=1
$$


Next we want to construct a function $\tilde{\varphi}$ such that (12) is satisfied. As an Ansatz we use the special Möbiustransformation

$$
\tilde{\varphi}(y)=\frac{\omega_{0} y}{1+\beta y}
$$

with $\beta \neq 0$ and $\tilde{\varphi}^{l_{1}}(y)=y$. For $1 \leq \nu \leq l_{1}-1$ we obtain

$$
\tilde{\varphi}^{\nu}(y)=\frac{\omega_{0}^{\nu} y}{1+\beta\left(1+\omega_{0}+\ldots+\omega_{0}^{\nu-1}\right) y} .
$$

For $\nu=l_{1}$ we obtain $\tilde{\varphi}^{l_{1}}(y)=y$, hence $\tilde{\varphi}$ is linearizable as well as $\psi$. Then we get

$$
1+w_{0}^{-1} \tilde{\varphi}^{\nu}(y)=1+w_{0}^{-1} \frac{\omega_{0} y}{1+\beta\left(1+\omega_{0}+\ldots+\omega_{0}^{\nu-1}\right) y}=\frac{1+\left[w_{0}^{-1} \omega_{0}^{\nu}+\beta\left(1+\omega_{0}+\ldots+\omega_{0}^{\nu-1}\right)\right] y}{1+\beta\left(1+\omega_{0}+\ldots+\omega_{0}^{\nu-1}\right) y} .
$$

Substituting this in equation (12) leads to

$$
\frac{1+w_{0}^{-1} y}{1} \cdot \frac{1+\left[w_{0}^{-1} \omega_{0}+\beta\right] y}{1+\beta y} \cdot \ldots \cdot \frac{1+\left[w_{0}^{-1} \omega_{0}^{l_{1}-1}+\beta\left(1+\omega_{0}+\ldots+\omega_{0}^{l_{1}-2}\right)\right] y}{1+\beta\left(1+\omega_{0}+\ldots \omega_{0}^{l_{1}-2}\right) y}=1
$$

If in each linear factor in the numerator of the left hand side of (13) the coefficient of $y$ is not zero, then the degree of the product is $l_{1}$ while the degree of the denominator is $l_{1}-1$ which contradicts (13). So it is necessary that $\beta$ is one of the numbers

$$
\begin{aligned}
\beta_{1} & =-w_{0}^{-1} \omega_{0} \\
\beta_{2} & =-w_{0}^{-1} \omega_{0}^{2}\left(1+\omega_{0}\right)^{-1} \\
& \vdots \\
\beta_{l_{1}-1} & =-w_{0}^{-1} \omega_{0}^{l_{1}-1}\left(1+\omega_{0}+\ldots+\omega_{0}^{l_{1}-2}\right)^{-1} .
\end{aligned}
$$

Hence for $1 \leq \mu \leq l_{1}-1$ we have

$$
\beta_{\mu}=-w_{0}^{-1}\left(1+\omega_{0}+\ldots+\omega_{0}^{\mu-1}\right)^{-1} \omega_{0}^{\mu}
$$

Next we have to show that this is sufficient. Therefore we number the fractions of (13) such that the numerators are $Z_{0}$ to $Z_{l_{1}-2}$ and the denominators are $N_{1}$ to $N_{l_{1}-2}$, for $N_{0}$ we have $N_{0}=1$. Then we choose a special $\beta_{\mu}$ for $1 \leq \mu \leq l_{1}-1$. Then it is true that $Z_{\nu}=N_{\nu-\mu}$ for $\nu \geq \mu$ and that $Z_{\nu}=N_{\nu+l_{1}-\mu}$ for $\nu \leq \mu$ hold. First we show that for $\nu \geq \mu$ we get $Z_{\nu}=N_{\nu-\mu}$. We obtain

$$
\begin{aligned}
Z_{\nu} & =1+\left[w_{0}^{-1} \omega_{0}^{\nu}+\left(-w_{0}^{-1}\left(1+\omega_{0}+\ldots+\omega_{0}^{\mu-1}\right)^{-1} \omega_{0}^{\mu}\right)\left(1+\omega_{0}+\ldots+\omega_{0}^{\nu-1}\right)\right] y \\
& =1+\left[w_{0}^{-1} \omega_{0}^{\nu}-w_{0}^{-1} \omega_{0}^{\mu}\left(1+\omega_{0}+\ldots+\omega_{0}^{\mu-1}\right)^{-1}\left(1+\omega_{0}+\ldots+\omega_{0}^{\nu-1}\right)\right] y .
\end{aligned}
$$

For $N_{\nu-\mu}$ we get

$$
N_{\nu-\mu}=1+\left(-w_{0}^{-1}\left(1+\omega_{0}+\ldots+\omega_{0}^{\mu-1}\right)^{-1} \omega_{0}^{\mu}\right)\left(1+\omega_{0}+\ldots+\omega_{0}^{\nu-\mu-1}\right)
$$

So it remains to show that

$$
\omega_{0}^{\nu}-\frac{\omega_{0}^{\mu}\left(1+\omega_{0}+\ldots+\omega_{0}^{\nu-1}\right)}{1+\omega_{0}+\ldots+\omega_{0}^{\mu-1}}=-\frac{\omega_{0}^{\mu}\left(1+\omega_{0}+\ldots+\omega_{0}^{\nu-\mu-1}\right)}{1+\omega_{0}+\ldots+\omega_{0}^{\mu-1}} .
$$


Expanding this equation leads to

$$
\omega_{0}^{\nu}+\omega_{0}^{\nu+1}+\ldots+\omega_{0}^{\nu+\mu-1}-\omega_{0}^{\mu}-\omega_{0}^{\mu+1}-\ldots-\omega_{0}^{\nu-1}-\omega_{0}^{\nu}-\ldots-\omega_{0}^{\mu+\nu-1}
$$

for the left hand side and to

$$
-\omega_{0}^{\mu}-\omega_{0}^{\mu+1}-\ldots-\omega_{0}^{\nu-1}
$$

for the right hand side. So everything cancels and hence we get $Z_{\nu}=N_{\nu-\mu}$ for $\nu \geq \mu$. Next we show that $Z_{\nu}=N_{\nu+l_{1}-\mu}$ for $\nu \leq \mu$. Like above we obtain

$$
Z_{\nu}=1+\left[w_{0}^{-1} \omega_{0}^{\nu}-w_{0}^{-1} \omega_{0}^{\mu}\left(1+\omega_{0}+\ldots+\omega_{0}^{\mu-1}\right)^{-1}\left(1+\omega_{0}+\ldots+\omega_{0}^{\nu-1}\right)\right] y
$$

for $Z_{\nu}$. The denominator $N_{\nu+l_{1}-\mu}$ is given by

$$
N_{\nu+l_{1}-\mu}=1+\left(-w_{0}^{-1} \omega_{0}^{\mu}\left(1+\omega_{0}+\ldots+\omega_{0}^{\mu-1}\right)^{-1}\left(1+\omega_{0}+\ldots+\omega_{0}^{\nu+l_{1}-\mu-1}\right)\right) y .
$$

After cancelling 1 and $w_{0}^{-1}$ one has to show that

$$
\omega_{0}^{\nu}-\frac{\omega_{0}^{\mu}\left(1+\omega_{0}+\ldots+\omega_{0}^{\nu-1}\right)}{1+\omega_{0}+\ldots+\omega_{0}^{\mu-1}}=-\frac{\omega_{0}^{\mu}\left(1+\omega_{0}+\ldots+\omega_{0}^{\nu+l_{1}-\mu-1}\right)}{1+\omega_{0}+\ldots \omega_{0}^{\mu-1}}
$$

is fulfilled. Expanding this equation leads to

$$
\omega_{0}^{\nu}+\omega_{0}^{\nu+1}+\ldots+\omega_{0}^{\mu}+\ldots+\omega_{0}^{\nu+\mu-1}-\omega_{0}^{\mu}-\omega_{0}^{\mu+1}-\ldots-\omega_{0}^{\nu+\mu-1}
$$

for the left hand side and hence only the expression

$$
\omega_{0}^{\nu}+\omega_{0}^{\nu+1}+\ldots+\omega_{0}^{\mu-1}
$$

remains. The right hand side is given by

$$
-\omega_{0}^{\mu}-\omega_{0}^{\mu+1}-\ldots-\omega_{0}^{\nu+l_{1}-1} .
$$

Therefore we have to consider the equation

$$
\omega_{0}^{\nu}\left(1+\omega_{0}+\ldots+\omega_{0}^{\mu-\nu-1}\right)=-\omega_{0}^{\nu}\left(\omega_{0}^{\mu-\nu}+\omega_{0}^{\mu-\nu+1}+\ldots+\omega_{0}^{l_{1}-1}\right)
$$

which is equivalent to

$$
1+\omega_{0}+\ldots+\omega_{0}^{\mu-\nu-1}=-\omega_{0}^{\mu-\nu}-\omega_{0}^{\mu-\nu+1}-\ldots-\omega_{0}^{l_{1}-1} .
$$

For a root of unity $\omega_{0}$ of order $l_{1}$ the equation $1+\omega_{0}+\ldots+\omega_{0}^{l_{1}-1}=0$ holds and hence (15) is fulfilled. Therefore our claim is valid which means that for every $\beta_{\mu}$ with $1 \leq \mu \leq l_{1}-1$ the corresponding solution is sufficient to solve (13).

In the next step we are looking for a special solution $U$ of $(3)\left(w_{0}+z^{k}\right) U(z)=U(\psi(z))$. Remember that our functions are given by

$$
\psi(z)=\frac{w_{0} z}{\left(1+\beta_{\mu} z^{k}\right)^{\frac{1}{k}}} .
$$

for $1 \leq \mu \leq l_{1}-1$. To show how one can compute a special solution we consider the special case $\beta_{2}=-w_{0}^{-1}(1+$ $\left.\omega_{0}\right)^{-1} \omega_{0}^{2}$ and we try to find a special solution for $\beta_{2}$. Therefore the function $\psi$ is given by $\psi(z)=\frac{w_{0} z}{\left(1+\beta_{2} z^{k}\right)^{\frac{1}{k}}}$. First we write $U(z)=z U^{(1)}(z)$ with $U^{(1)}(z)=1+\ldots$. Then (3) is equivalent to

$$
w_{0}\left(1+w_{0}^{-1} z^{k}\right) z U^{(1)}(z)=\psi(z) U^{(1)}(\psi(z))
$$


and this is equivalent to

$$
w_{0} z\left(1+w_{0}^{-1} z^{k}\right) U^{(1)}(z)=w_{0} z\left(1+\beta_{2} z^{k}\right)^{-\frac{1}{k}} U^{(1)}(\psi(z)) .
$$

This leads to

$$
\left(1+w_{0}^{-1} z^{k}\right)\left(1+\beta_{2} z^{k}\right)^{\frac{1}{k}} U^{(1)}(z)=U^{(1)}(\psi(z)) .
$$

Then we use a new Ansatz, so we consider $U^{(1)}(z)=\left(1+w_{0}^{-1} z^{k}\right)^{\alpha_{1}} U^{(2)}(z)$ and after substituting this expression in (16) we get

$$
\left(1+w_{0}^{-1} z^{k}\right)^{\alpha_{1}+1}\left(1+\beta_{2} z^{k}\right)^{\frac{1}{k}} U^{(2)}(z)=\left(1+w_{0}^{-1} \psi(z)^{k}\right)^{\alpha_{1}} U^{(2)}(\psi(z))
$$

or the equivalent expression

$$
\left(1+w_{0}^{-1} z^{k}\right)^{\alpha_{1}+1}\left(1+\beta_{2} z^{k}\right)^{\frac{1}{k}} U^{(2)}(z)=\left(1+w_{0}^{-1} \frac{\omega_{0} z^{k}}{1+\beta_{2} z^{k}}\right)^{\alpha_{1}} U^{(2)}(\psi(z)) .
$$

This leads to

$$
\left(1+w_{0}^{-1} z^{k}\right)^{\alpha_{1}+1}\left(1+\beta_{2} z^{k}\right)^{\frac{1}{k}+\alpha_{1}} U^{(2)}(z)=\left(1+\left(\beta_{2}+w_{0}^{-1} \omega_{0}\right) z^{k}\right)^{\alpha_{1}} U^{(2)}(\psi(z)) .
$$

Then again a new Ansatz is needed and by $U^{(2)}(z)=\left(1+\left(\beta_{2}+w_{0}^{-1} \omega_{0}\right) z^{k}\right)^{\alpha_{1}} U^{(3)}(z)$ we describe this new one. Substituting this in (17) leads to

$$
\begin{aligned}
& \left(1+w_{0}^{-1} z^{k}\right)^{\alpha_{1}+1}\left(1+\beta_{2} z^{k}\right)^{\frac{1}{k}+\alpha_{1}}\left(1+\left(\beta_{2}+w_{0}^{-1} \omega_{0}\right) z^{k}\right)^{\alpha_{1}} U^{(3)}(z)= \\
& \left(1+\left(\beta_{2}+w_{0}^{-1} \omega_{0}\right) z^{k}\right)^{\alpha_{1}}\left(1+\left(\beta_{2}+w_{0}^{-1} \omega_{0}\right) \psi(z)^{k}\right)^{\alpha_{1}} U^{(3)}(\psi(z)) .
\end{aligned}
$$

This is equivalent to

$$
\left(1+w_{0}^{-1} z^{k}\right)^{\alpha_{1}+1}\left(1+\beta_{2} z^{k}\right)^{\frac{1}{k}+\alpha_{1}} U^{(3)}(z)=\left(1+\left(\beta_{2}+w_{0}^{-1} \omega_{0}\right) \frac{\omega_{0} z^{k}}{1+\beta_{2} z^{k}}\right)^{\alpha_{1}} U^{(3)}(\psi(z)) .
$$

Hence we get

$$
\begin{aligned}
\left(1+w_{0}^{-1} z^{k}\right)^{\alpha_{1}+1}\left(1+\beta_{2} z^{k}\right)^{\frac{1}{k}+\alpha_{1}} U^{(3)}(z) & =\left(\frac{1+\beta_{2} z^{k}+\beta_{2} \omega_{0} z^{k}+w_{0}^{-1} \omega_{0}^{2} z^{k}}{1+\beta_{2} z^{k}}\right)^{\alpha_{1}} U^{(3)}(\psi(z)) \\
& =\left(\frac{1+\left[\beta_{2}\left(1+\omega_{0}\right)+w_{0}^{-1} \omega_{0}^{2}\right] z^{k}}{1+\beta_{2} z^{k}}\right)^{\alpha_{1}} U^{(3)}(\psi(z)) \\
& =\left(\frac{1}{1+\beta_{2} z^{k}}\right)^{\alpha_{1}} U^{(3)}(\psi(z))
\end{aligned}
$$

because the condition which the expression $\beta_{2}$ has to fulfill is $\beta_{2}\left(1+\omega_{0}\right)+w_{0}^{-1} \omega_{0}^{2}=0$. Hence the following equation remains

$$
\left(1+w_{0}^{-1} z^{k}\right)^{\alpha_{1}+1}\left(1+\beta_{2} z^{k}\right)^{\frac{1}{k}+2 \alpha_{1}} U^{(3)}(z)=U^{(3)}(\psi(z))
$$

and so we need a last Ansatz. We choose $U^{(3)}(z)=\left(1+\delta z^{k}\right)^{\alpha_{2}}$ and substituting this expression in (18) we get for the right hand side of (18)

$$
U^{(3)}(\psi(z))=\left(1+\delta \frac{\omega_{0} z^{k}}{1+\beta_{2} z^{k}}\right)^{\alpha_{2}}=\left(\frac{1+\left(\beta_{2}+\delta \omega_{0}\right) z^{k}}{1+\beta_{2} z^{k}}\right)^{\alpha_{2}} .
$$


Therefore (18) is equivalent to

$$
\left(1+w_{0}^{-1} z^{k}\right)^{\alpha_{1}+1}\left(1+\beta_{2} z^{k}\right)^{\frac{1}{k}+2 \alpha_{1}+\alpha_{2}}\left(1+\delta z^{k}\right)^{\alpha_{2}}=\left(1+\left(\beta_{2}+\delta \omega_{0}\right) z^{k}\right)^{\alpha_{2}} .
$$

Hence we obtain $\delta=\beta_{2}+\delta \omega_{0}$ which is equaivalent to $\delta=\left(1-\omega_{0}\right)^{-1} \beta_{2}$. Also $\left(1+w_{0}^{-1} z^{k}\right)^{\alpha_{1}+1}(1+$ $\left.\beta_{2} z^{k}\right)^{\frac{1}{k}+2 \alpha_{1}+\alpha_{2}}=1$ has to be satisfied and this leads to $\alpha_{1}=-1$ and $\alpha_{2}=2-\frac{1}{k}$. Then the special solution for $\beta_{2}$ is given by

$$
U(z)=z\left(1+w_{0}^{-1} z^{k}\right)^{-1}\left(1+\left(\beta_{2}+w_{0}^{-1} \omega_{0}\right) z^{k}\right)^{-1}\left(1+\left(1-\omega_{0}\right)^{-1} \beta_{2} z^{k}\right)^{2-\frac{1}{k}}
$$

which we obtain after putting our results together.

These calculations motivate to state that a special solution $U$ of equation (3) for a $\beta_{\mu}$ with $1 \leq \mu \leq l_{1}-1$ has the representation

$$
U(z)=z \prod_{\nu=0}^{\mu-1}\left(1+\left[\beta_{\mu}\left(1+\omega_{0}+\ldots+\omega_{0}^{\nu-1}\right)+w_{0}^{-1} \omega_{0}^{\nu}\right] z^{k}\right)^{-1}\left(1+\beta_{\mu}\left(1-\omega_{0}\right)^{-1} z^{k}\right)^{\mu-\frac{1}{k}} .
$$

To proof this we have to substitute $U(z)$ in $(3)$. Then the left hand side of $(3)$ is

$$
\begin{aligned}
\left(w_{0}+z^{k}\right) U(z)= & w_{0}\left(1+w_{0}^{-1} z^{k}\right) z \prod_{\nu=0}^{\mu-1}\left(1+\left[\beta_{\mu}\left(1+\omega_{0}+\ldots+\omega_{0}^{\nu-1}\right)+w_{0}^{-1} \omega_{0}^{\nu}\right] z^{k}\right)^{-1} \\
& \left(1+\beta_{\mu}\left(1-\omega_{0}\right)^{-1} z^{k}\right)^{\mu-\frac{1}{k}} \\
= & w_{0} z\left(1+w_{0}^{-1} z^{k}\right)^{1-1} \prod_{\nu=1}^{\mu-1}\left(1+\left[\beta_{\mu}\left(1+\omega_{0}+\ldots+\omega_{0}^{\nu-1}\right)+w_{0}^{-1} \omega_{0}^{\nu}\right] z^{k}\right)^{-1} \\
& \left(1+\beta_{\mu}\left(1-\omega_{0}\right)^{-1} z^{k}\right)^{\mu-\frac{1}{k}}
\end{aligned}
$$

The right hand side of (3) is given by

$$
\begin{aligned}
U(\psi(z))= & \psi(z) \prod_{\nu=0}^{\mu-1}\left(1+\left[\beta_{\mu}\left(1+\omega_{0}+\ldots+\omega_{0}^{\nu-1}\right)+w_{0}^{-1} \omega_{0}^{\nu}\right] \psi(z)^{k}\right)^{-1} \\
& \left(1+\beta_{\mu}\left(1-\omega_{0}\right)^{-1} \psi(z)^{k}\right)^{\mu-\frac{1}{k}} \\
= & w_{0} z\left(1+\beta_{\mu} z^{k}\right)^{-\frac{1}{k}} \prod_{\nu=0}^{\mu-1}\left(1+\left[\beta_{\mu}\left(1+\omega_{0}+\ldots+\omega_{0}^{\nu-1}\right)+w_{0}^{-1} \omega_{0}^{\nu}\right] \frac{\omega_{0} z^{k}}{1+\beta_{\mu} z^{k}}\right)^{-1} \\
& \left(1+\beta_{\mu}\left(1-\omega_{0}\right)^{-1} \frac{\omega_{0} z^{k}}{1+\beta_{\mu} z^{k}}\right)^{\mu-\frac{1}{k}} \\
= & w_{0} z\left(1+\beta_{\mu} z^{k}\right)^{-\frac{1}{k}+\mu-\mu+\frac{1}{k}} \prod_{\nu=0}^{\mu-1}\left(1+\beta_{\mu} z^{k}+\left[\beta_{\mu}\left(1+\omega_{0}+\ldots+\omega_{0}^{\nu-1}\right)+w_{0}^{-1} \omega_{0}^{\nu}\right]\right. \\
& \left.\omega_{0} z^{k}\right)^{-1}\left(1+\beta_{\mu} z^{k}+\beta_{\mu}\left(1-\omega_{0}\right)^{-1} \omega_{0} z^{k}\right)^{\mu-\frac{1}{k}} .
\end{aligned}
$$

The last term of the right hand side leads to

$$
\begin{aligned}
\left(1+\beta_{\mu} z^{k}+\beta_{\mu}\left(1-\omega_{0}\right)^{-1} \omega_{0} z^{k}\right)^{\mu-\frac{1}{k}} & =\left(1+\beta_{\mu}\left(\left(1+\left(1-\omega_{0}\right)^{-1}\right) \omega_{0}\right) z^{k}\right)^{\mu-\frac{1}{k}} \\
& =\left(1+\beta_{\mu}\left(1-\omega_{0}\right)^{-1} z^{k}\right)^{\mu-\frac{1}{k}}
\end{aligned}
$$


and hence this terms and the term $w_{0} z$ cancel in the equation. For $1 \leq n \leq \mu-1$ the $n-t h$ factor of the product of the left hand side of the equation is given by

$$
\left(1+\left[\beta_{\mu}\left(1+\omega_{0}+\ldots+\omega_{0}^{n-1}\right)+w_{0}^{-1} \omega_{0}^{n}\right] z^{k}\right)^{-1}
$$

and the $(n-1)-t h$ factor of the right hand side by

$$
\left(1+\beta_{\mu} z^{k}+\left[\beta_{\mu}\left(1+\omega_{0}+\ldots+\omega_{0}^{n-2}\right)+w_{0}^{-1} \omega_{0}^{n-1}\right] \omega_{0} z^{k}\right)^{-1}
$$

Now it is easy to see that these expressions are the same. It remains to consider the $(\mu-1)-t h$ factor of the right hand side of the equation. We obtain

$$
\left(1+\beta_{\mu} z^{k}+\left[\beta_{\mu}\left(1+\omega_{0}+\ldots+\omega_{0}^{\mu-2}\right)+w_{0}^{-1} \omega_{0}^{\mu-1}\right] \omega_{0} z^{k}\right)^{-1}=\left(1+\left[\beta_{\mu}\left(1+\omega_{0}+\ldots+\omega_{0}^{\mu-1}\right)+w_{0}^{-1} \omega_{0}^{\mu}\right] z^{k}\right)^{-1}
$$

and this equals 1 because $\beta_{\mu}$ has to fulfill $\beta_{\mu}\left(1+\omega_{0}+\ldots+\omega_{0}^{\mu-1}\right)+w_{0}^{-1} \omega_{0}^{\mu}=0$. Hence the function $U(z)$ which we obtain is a special solution of $(3)\left(w_{0}+z^{k}\right) U(z)=U(\psi)$.

In the next step we want to find a function $S$ such that we can linearize the function $\psi(z)=\frac{w_{0} z}{\left(1+\beta_{\mu} z^{k}\right)^{\frac{1}{k}}}$ that means such that $S(\psi(z))=w_{0} S(z)$ holds. To construct such a $S$ we can use similar calculations as above. For $1 \leq \mu \leq l_{1}-1$ we get

$$
S(z)=z\left(1+\beta_{\mu}\left(1-\omega_{0}\right)^{-1} z^{k}\right)^{-\frac{1}{k}} .
$$

It is easy to show that the linearization equation holds, we obtain

$$
w_{0} S(z)=w_{0} z\left(1+\beta_{\mu}\left(1-\omega_{0}\right)^{-1} z^{k}\right)^{-\frac{1}{k}}
$$

for the right hand side and

$$
\begin{aligned}
S(\psi(z)) & =\psi(z)\left(1+\beta_{\mu}\left(1-\omega_{0}\right)^{-1} \psi(z)^{k}\right)^{-\frac{1}{k}} \\
& =\frac{w_{0} z}{\left(1+\beta_{\mu} z^{k}\right)^{\frac{1}{k}}}\left(1+\beta_{\mu}\left(1-\omega_{0}\right)^{-1} \frac{\omega_{0} z^{k}}{\left(1+\beta_{\mu} z^{k}\right)}\right)^{-\frac{1}{k}} \\
& =w_{0} z\left(1+\beta_{\mu} z^{k}\right)^{-\frac{1}{k}+\frac{1}{k}}\left(1+\beta_{\mu} z^{k}+\beta_{\mu}\left(1-\omega_{0}\right)^{-1} \omega_{0} z^{k}\right)^{-\frac{1}{k}} \\
& =w_{0} z\left(1+\beta_{\mu}\left(1+\left(1-\omega_{0}\right)^{-1} \omega_{0}\right) z^{k}\right)^{-\frac{1}{k}} \\
& =w_{0} z\left(1+\beta_{\mu}\left(1-\omega_{0}\right)^{-1} z^{k}\right)^{-\frac{1}{k}}
\end{aligned}
$$

for the left hand side and hence the function $S$ is a linearization of $\psi$. Now we are interested in the general solution $U$ of $(3)\left(w_{0}+z^{k}\right) U(z)=U(\psi(z))$ with $\psi(z)=\frac{w_{0} z}{\left(1+\beta_{\mu} z^{k}\right)^{\frac{1}{k}}}, 1 \leq \mu \leq l_{1}-1$. The general solution has the representation $U(z)=U^{0}(z) W(z)$. If $W(z)=\frac{U_{1}(z)}{U_{2}(z)}$ for two solutions $U_{1}$ and $U_{2}$ of $(3)$ we get

$$
\begin{aligned}
W(z)=W\left(\frac{w_{0} z}{\left(1+\beta_{\mu} z^{k}\right)^{\frac{1}{k}}}\right) & \Leftrightarrow W(z)=W\left(S^{-1}\left(w_{0} S(z)\right)\right) \\
& \Leftrightarrow W\left(S^{-1}(z)\right)=W\left(S^{-1}\left(w_{0} z\right)\right) .
\end{aligned}
$$

Defining the function $\Phi=W \circ S^{-1}$ we obtain

$$
\Phi(z)=\Phi\left(w_{0} z\right)
$$


That means the function $\Phi$ is invariant with respect to $w_{0}$ and hence we have the representation $\Phi(z)=$ $1+\phi_{l} z^{l}+\phi_{2 l} z^{2 l}+\ldots$. Introducing the function $\Phi^{\star}$ such that $\Phi(z)=\Phi^{\star}\left(z^{l}\right)$ and $\Phi^{\star}(z)=1+\ldots$ holds, leads to $W(z)=\Phi^{\star}\left(S(z)^{l}\right)$. Finally we receive

$$
\begin{aligned}
U(z)= & z \prod_{\nu=0}^{\mu-1}\left(1+\left[\beta_{\mu}\left(1+\omega_{0}+\ldots+\omega_{0}^{\nu-1}\right)+w_{0}^{-1} \omega_{0}^{\nu}\right] z^{k}\right)^{-1} \\
& \left(1+\beta_{\mu}\left(1-\omega_{0}\right)^{-1} z^{k}\right)^{\mu-\frac{1}{k}} \Phi^{\star}\left(\left[z\left(1+\beta_{\mu}\left(1-\omega_{0}\right)^{-1} z^{k}\right)^{-\frac{1}{k}}\right]^{l}\right)
\end{aligned}
$$

as the general solution $U$ of $(3)\left(w_{0}+z^{k}\right) U(z)=U(\psi(z))$.

Now we summarize this situation. Let $\psi$ be linearizable and hence also the function $\tilde{\varphi}$ is, $\psi(z)=w_{0} z+\ldots$ and the relation $\psi(z)^{k}=\tilde{\varphi}\left(z^{k}\right)$, where $w_{0}$ is a primitive root of unity of order $l \geq 2$ holds. We define $\omega_{0}:=w_{0}^{k}$ and hence this is a primitive root of unity of order $l_{1}=\frac{l}{\operatorname{gcd}(k, l)}$.

Hence we have proven the following theorem.

Theorem 2.1. Let $\tilde{\varphi}$ be given by

$$
\tilde{\varphi}(y)=\frac{\omega_{0} y}{1+\beta y}
$$

where $\beta$ is one of the complex numbers $\beta_{\mu}$ given by (14). Then the general solution $U$ of the linear functional equation (3) $\left(w_{0}+z^{k}\right) U(z)=U(\psi(z))$ is given by

$$
\begin{aligned}
U(z)= & z \prod_{\nu=0}^{\mu-1}\left(1+\left[\beta_{\mu}\left(1+\omega_{0}+\ldots+\omega_{0}^{\nu-1}\right)+w_{0}^{-1} \omega_{0}^{\nu}\right] z^{k}\right)^{-1} \\
& \left(1+\beta_{\mu}\left(1-\omega_{0}\right)^{-1} z^{k}\right)^{\mu-\frac{1}{k}} \Phi^{\star}\left(\left[z\left(1+\beta_{\mu}\left(1-\omega_{0}\right)^{-1} z^{k}\right)^{-\frac{1}{k}}\right]^{l}\right)
\end{aligned}
$$

where the function $\Phi^{\star}$ is arbitrary and $\Phi^{\star}(z) \in \mathbb{C} \llbracket z \rrbracket$. The $\beta_{\mu}$ 's with $1 \leq \mu \leq l_{1}-1$ are given by (14).

\section{LOCAL ANALYTIC SOLUTIONS}

Theorem 3.1. Let $\tilde{\varphi}$ be linearizable and convergent in a sufficiently small neighbourhood of zero. Then the equation

$$
g\left(w_{0} z+z g(z)\right)=\tilde{\varphi}(g(z))
$$

has convergent solutions $g$ and hence also the generalized Dhombres functional equation

$$
f(z f(z))=\varphi(f(z))
$$

has non constant convergent solutions $f$ with $f(0)=w_{0}$.

Proof. It is known that (2) can be written as linear functional equation

$$
\left(w_{0}+z^{k}\right) U(z)=U(\psi(z))
$$

with $\psi(z)^{k}=\varphi\left(z^{k}\right)$ and $U(z)=u_{1} z+\ldots$. This is the same as

$$
\frac{z\left(w_{0}+z^{k}\right)}{\psi(z)} U^{\star}(z)=U^{\star}(\psi(z))
$$


with $U(z)=u_{1} z U^{\star}(z)=u_{1} z(1+\ldots)$. Hence it is possible to use the formal logarithm which leads to

$$
\operatorname{Ln} \frac{z\left(w_{0}+z^{k}\right)}{\psi(z)}+X^{\star}(z)=X^{\star}(\psi(z))
$$

with $X^{\star}=\operatorname{Ln} U^{\star}$. Then it is known that $\operatorname{Ln} \frac{z\left(w_{0}+z^{k}\right)}{\psi(z)} \in \mathbb{C} \llbracket z^{k} \rrbracket$ and that $\operatorname{Ln} \frac{z\left(w_{0}+z^{k}\right)}{\psi(z)}$ is convergent. So (2) is solvable if and only if (19) has a solution $X^{\star}(z)=\tilde{X}\left(z^{k}\right)$. Using $y=z^{k}$ and $A(y)=\operatorname{Ln} \frac{z\left(w_{0}+z^{k}\right)}{\psi(z)}$ we obtain

$$
A(y)+\tilde{X}(y)=\tilde{X}(\tilde{\varphi}(y)) .
$$

Then $\tilde{\varphi}$ is linearizable and hence, see [5], there exists a convergent $R$ such that $\tilde{\varphi}(y)=R^{-1}\left(\omega_{0} R(y)\right)$ with $\omega_{0}=w_{0}^{k}$ holds. Writing $A \circ R^{-1}=B$ and $\tilde{X} \circ R^{-1}=X$ leads to

$$
B(y)+X(y)=X\left(\omega_{0} y\right)
$$

with a convergent series $B$. Let $B(y)=\sum_{\nu \geq 1} \beta_{\nu} y^{\nu}$ and $X(y)=\sum_{\nu \geq 1} \xi_{\nu} y^{\nu}$ then we get $X(\psi(y))-X(y)=$ $\sum_{\substack{\nu \geq 1 \\ \nu \neq 0\left(\bmod l_{1}\right)}} \xi_{\nu}\left(\omega_{0}^{\nu}-1\right) y^{\nu}$ where $l_{1}$ denotes the order of $\omega_{0}$. Therefore we obtain

$$
B(y)=\sum_{\substack{\nu \geq 1 \\ \nu \neq 0\left(\bmod l_{1}\right)}} \xi_{\nu}\left(\omega_{0}^{\nu}-1\right) y^{\nu}
$$

and hence a special solution of (20) is given by

$$
X_{0}(y)=\sum_{\substack{\nu \geq 1 \\ \nu \neq 0\left(\bmod l_{1}\right)}} \frac{\beta_{\nu}}{\left(\omega_{0}^{\nu}-1\right)} y^{\nu}
$$

and this solution is convergent since there exists $r_{0}>0$, such that $\left|\omega_{0}^{\nu}-1\right|^{-1} \leq r_{0}$ for all $\nu$ with $\nu \not \equiv 0\left(\bmod l_{1}\right)$. Now we choose the coefficients $\xi_{\nu l_{1}}$ with $\nu \geq 1$ such that the series

$$
X(y)=X_{0}(y)+\sum_{\nu \geq 1} \xi_{\nu l_{1}} y^{\nu l_{1}}
$$

is also convergent. It is possible to reverse these calculations and hence there exist local analytic solutions $g$ of the transformed generalized Dhombres functional equation (2).

\section{REFERENCES}

[1] Jean Dhombres, Some Aspects of Functional Equations, Chulalongkorn University Press, Bangkok, 1979.

[2] Harald Fripertinger, Ludwig Reich, On a Linear Functional Equation for Formal Power Series, Sitzungsber. Österr. Akad. Wiss. Wien, Math.-nat Kl. Abt. II 210: 85-134, 2001.

[3] Ludwig Reich, Jaroslav Smítal, Marta Štefánková, Local Analytic Solutions of the Generalized Dhombres Functional Equations I, Sitzungsber. Österr. Akad. Wiss. Wien, Math.-nat Kl. Abt. II 214: 3-25, 2005.

[4] Ludwig Reich, Jaroslav Smítal, Marta Štefánková, Local Analytic Solutions of the Generalized Dhombres Functional Equations II, Journal of Mathematical Analysis and Applications 355: 821-829, 2009.

[5] Ludwig Reich, A remark on Schröder's equation in the complex domain, in preparation. 